\title{
A síndrome da imunodeficiência humana adquirida como fator na redução da espessura e aumento do colágeno nas trabéculas ósseas de adultos e idosos
}

The acquired human immunodeficiency syndrome as a factor in reducing the thickness and increasing collagen in bone trabeculae of adults and the elderly

El síndrome de inmunodeficiencia humana adquirida como factor de reducción del grosor y aumento del colágeno en las trabéculas óseas de adultos y ancianos

Flávio José Pereira de Almeida Ferreira ORCID: https://orcid.org/0000-0001-6523-0569 Universidade Federal do Triângulo Mineiro, Brasil E-mail: flavio-4040@hotmail.com

Fabrizio Antonio Gomide Cardoso ORCID: https://orcid.org/0000-0001-7520-2879 Universidade Federal do Triângulo Mineiro, Brasil E-mail: fabriziouftm@gmail.com

Vandair Gonçalves Pereira

ORCID: https://orcid.org/0000-0001-6945-5503

Universidade Federal do Triângulo Mineiro, Brasil E-mail: c.bvandair@yahoo.com.br

Gabriel Antonio Nogueira Nascentes

ORCID: https://orcid.org/0000-0002-8934-6619

Universidade Federal do Triângulo Mineiro, Brasil E-mail: gabrielnog@yahoo.com.br

Valéria Silva Monteiro

ORCID: https://orcid.org/0000-0003-4208-0654 Universidade Federal do Triângulo Mineiro, Brasil E-mail valeriasmonteiro69@gmail.com

Ana Paula Espindula

ORCID: https://orcid.org/0000-0002-9282-4482 Universidade Federal do Triângulo Mineiro, Brasil

E-mail: anapaulaespindula@yahoo.com.br

\section{Resumo}

A Síndrome da Imunodeficiência Humana Adquirida (SIDA) onera a sociedade e representa um dos maiores desafios da saúde mundial em decorrência da sua gravidade, disseminação e desigualdade ao acesso a terapia antirretroviral. Objetivo: analisar a espessura das trabéculas ósseas e a densidade de colágeno total de fragmentos de autópsias da medula óssea de indivíduos com SIDA. Metodologia: estudo observacional retrospectivo, analítico e quantitativo, realizado a partir de 60 fragmentos da medula óssea de indivíduos autopsiados, com idade entre 26 e 71 anos, divididos em quatro grupos, adultos e idosos com e sem SIDA. Para as análises histomorfométricas foram utilizadas as técnicas de histomorfometria com microscopia de luz comum para análise quantitativa da espessura e técnica de microscopia com luz polarizada para avaliação do colágeno total nas trabéculas ósseas. Resultados: os grupos de adultos e de idosos com SIDA apresentaram diminuição significativa na espessura das trabéculas ósseas em comparação aos grupos de adultos e de idosos sem SIDA $(p<0,001)$. Na avaliação do colágeno os grupos de adultos e de idosos com SIDA apresentaram aumento significativo do colágeno total $(\mathrm{p}<0,001)$ em comparação aos grupos sem SIDA. Na comparação entre os indivíduos adultos e idosos com SIDA houve aumento significativo do colágeno total no grupo de adultos $(\mathrm{p}=0,041)$. Como resultado complementar, o índice de massa corporal entre os grupos com a SIDA apresentou diminuição significativa no grupo de idosos $(\mathrm{p}=0,040)$ em comparação ao grupo de adultos. Conclusão: o fator SIDA influencia a diminuição da espessura das trabéculas ósseas e favorece o aumento do colágeno total em adultos e idosos sendo fator importante na avaliação da massa óssea, principalmente em indivíduos com mais de 50 anos que apresentam maior perda da matriz orgânica.

Palavras chave: Anatomia e histologia; Colágeno; Osso trabecular; Síndrome de imunodeficiência adquirida.

\section{Abstract}

The Acquired Human Immunodeficiency Syndrome (AIDS) burdens society and represents one of the greatest challenges of global health due to the severity, dissemination and inequality in access to antiretroviral therapy. 
Objective: to analyze the thickness of the bone trabeculae and the total collagen density of fragments from bone marrow autopsies with AIDS deficiency. Methodology: retrospective observational, analytical and quantitative study, carried out from 60 bone marrow fragments from autopsied patients, aged between 26 and 71 years, divided into four groups, adults and elderly with and without AIDS. For the histomorphometric analysis, histomorphometric techniques with common light microscopy were used for quantitative analysis of the thickness and microscopy technique with polarized light to evaluate the total collagen in the bone trabeculae. Results: Adult and elderly groups with dissipation AIDS decreased in trabecular bone thickness compared to groups of adults and elderly without AIDS (p<0.001). In the assessment of collagen in groups of adults and elderly people with AIDS, there was a significant increase in total collagen $(\mathrm{p}<0.001)$ compared to groups without AIDS. When comparing adults and elderly people with AIDS, there was a significant increase in total collagen in the adult group $(p=0.041)$. As a complementary result, the body mass index among the groups with AIDS decreased, decreasing in the elderly group $(p=0.040)$ compared to the adult group. Conclusion: the AIDS factor influences the decrease in the thickness of the bone trabeculae and favors the increase in total collagen in adults and the elderly, being an important factor in the assessment of bone mass, especially in those aged over 50 years who present the greatest loss of organic matrix.

Keywords: Acquired immunodeficiency syndrome; Anatomy e histology; Cancellous bone; Collagen.

\section{Resumen}

El Síndrome de Inmunodeficiencia Humana Adquirida (SIDA) agobia a la sociedad y representa uno de los mayores desafíos en la salud mundial por la gravedad, difusión y desigualdad en el acceso a la terapia antirretroviral. Objetivo: analizar el grosor de las trabéculas óseas y la densidad de colágeno total de fragmentos de autopsias de médula ósea con deficiencia de SIDA. Metodología: estudio retrospectivo observacional, analítico y cuantitativo, realizado a partir de 60 fragmentos de médula ósea de pacientes autopsiados, con edades entre 26 y 71 años, divididos en cuatro grupos, adultos y ancianos con y sin sida. Para los análisis histomorfométricos se utilizaron técnicas histomorfométricas con microscopía óptica común para el análisis cuantitativo del espesor y técnica de microscopía con luz polarizada para evaluar el colágeno total en las trabéculas óseas. Resultados: Los grupos de adultos y ancianos con SIDA de disipación disminuyeron en el grosor del hueso trabecular en comparación con los grupos de adultos y ancianos sin SIDA ( $p<0,001)$. En la evaluación del colágeno en grupos de adultos y ancianos con sida, hubo un aumento significativo del colágeno total $(\mathrm{p}<0,001)$ en comparación con los grupos sin sida. Al comparar adultos y ancianos con SIDA, hubo un aumento significativo en el colágeno total en el grupo de adultos $(\mathrm{p}=0.041)$. Como resultado complementario, el índice de masa corporal entre los grupos con sida disminuyó, disminuyendo en el grupo de ancianos $(\mathrm{p}=0.040)$ en comparación con el grupo de adultos. Conclusión: el factor SIDA influye en la disminución del grosor de las trabéculas óseas y favorece el aumento del colágeno total en adultos y ancianos, siendo un factor importante en la valoración de la masa ósea, especialmente en los mayores de 50 años que presentan la mayor Pérdida de matriz orgánica.

Palabras clave: Anatomía e histlogia; Colágeno; Hueso esponjoso; Síndrome de inmunodeficiencia adquirida.

\section{Introdução}

A Síndrome da Imunodeficiência Adquirida (SIDA) foi reconhecida em meados de 1981, nos Estados Unidos da América, a partir da identificação de pneumonia em cinco pacientes adultos jovens do gênero masculino, homossexuais no estado da Califórnia (Gottlieb, et al., 2021). Posteriormente, em abril de 1985 foi identificado que infecção pelo vírus da imunodeficiência humana (VIH) ocorria na maioria dos casos (kaslow, et al., 1987).

Desde os primeiros casos de infecção VIH, 78 milhões de pessoas foram infectadas e 35 milhões morreram de doenças relacionadas à SIDA no mundo (Unaids, 2020). No Brasil, em 2019, foram diagnosticados 41.909 novos casos de VIH e 37.308 casos de SIDA. A contabilizar nos últimos 40 anos, entre a década de 1980 e junho de 2020, 1.011.617 casos de SIDA foram detectados no país. Desde o início da epidemia de SIDA até 31 de dezembro de 2019, foram notificados no Brasil 349.784 óbitos tendo o VIH/SIDA como causa básica 4 (Secretaria de vigilância em saúde, 2020).

De 1987 até os dias de hoje a indústria farmacêutica vem trabalhando para aperfeiçoar cada vez mais os medicamentos da terapia antirretroviral (TARV), proporcionando aos pacientes com SIDA maior expectativa de vida e redução do risco de transmissão do VIH a pessoas não infectadas, entretanto indivíduos com VIH podem ter doenças não transmissíveis relacionadas (Odden, et al., 2007) ou não à idade (Triant, et al., 2007) mais precocemente, que pessoas não infectadas, incluindo doenças ósseas (Starup et al., 2020). 
O esqueleto humano adulto é composto por osso cortical e osso trabecular. O osso cortical também chamado de compacto circunda o espaço medular formando a camada sólida mais externa, enquanto o osso trabecular também chamado de osso esponjoso é composto por uma rede em formato lamelar semelhante a um favo de mel de placas trabeculares e hastes intercaladas no qual as fibrilas de colágeno são depositadas no compartimento da medula óssea. O mecanismo pelo qual os osteoblastos depositam as fibrilas de colágeno em padrão lamelar permanece desconhecido, mas essa orientação confere resistência ao osso (Baig \& Bacha, 2021; Clarke, 2008). A maior área de superfície facilita o osso trabecular ter maior atividade metabólica em comparação com o osso cortical, embora osso cortical seja capaz de suportar maior parte da carga o osso trabecular contribui e serve para carregar e compartilhar tensões. As trabéculas individuais de humanos têm espessura e formato que variam de hastes cilíndricas a placas achatadas e apresentam número variável em qualquer local ósseo determinado pela relação entre o tamanho das trabéculas (espessura) e sua quantidade no osso (Zaidi, et al., 2020).

Análises do osso trabecular em indivíduos autopsiados com SIDA, envolvendo a quantificação do colágeno são escassos na literatura (Dias, et al., 2015). Desse modo, é importante a realização de estudos que investiguem sua relação com a matriz orgânica e inorgânica do osso. Sendo assim, surge a hipótese desse estudo, a SIDA é um fator capaz de interferir na estrutura do osso trabecular e índice de massa corporal (IMC) em humanos. Nesse contexto, o presente estudo teve como objetivo analisar a espessura das trabéculas ósseas de fragmentos de medula óssea dos indivíduos adultos e idosos, com e sem SIDA, analisar a densidade de colágeno total das trabéculas ósseas dos indivíduos adultos e idosos, com e sem SIDA, analisar se o fator SIDA influenciou o IMC de adultos e idosos.

\section{Metodologia}

Estudo observacional retrospectivo, analítico e quantitativo com seres humanos autopsiados, aprovado pelo Comitê de Ética em Pesquisa- CEP sob protocolo de $\mathrm{n}^{\circ} 1.692 .900$. No estudo foram selecionados fragmentos da medula óssea do osso esterno coletados, no período de 1990 a 2018, a partir de laudos de autópsias armazenadas na disciplina de patologia geral da Universidade Federal do triângulo Mineiro em Uberaba, Minas Gerais, Brasil.

Para ser incluído no estudo os fragmentos foram selecionados de laudos com diagnóstico de SIDA. Para o diagnóstico foi utilizado os seguintes critérios: contagem de linfócitos TCD4 abaixo de 200 células $/ \mathrm{mm}^{3}$ ou a evidência de pelo menos uma das doenças definidoras no momento da autópsia, tais como, candidíase de esôfago, carcinoma invasivo de colo uterino, citomegalovirose, criptococose extrapulmonar, criptosporidiose intestinal, sarcoma de Kaposi, histoplamose disseminada, isosporidiose intestinal, leucoencefalopatia multifocal e ou linfoma não Hodgkin, tuberculose pulmonar disseminada e toxoplasmose de órgãos internos (Silva, 2019; Yarchoan \& Uldrick, 2018). Informações como, idade, cor, sexo e IMC também foram coletados dos prontuários.

Foram excluídos do estudo condições identificadas nos prontuários que pudesse alterar o IMC ou a medula óssea como, anasarca, nefropatias, doenças hepáticas, síndrome mielodisplásica, doenças mieloproliferativas, pacientes com anomalias congênitas ou cromossômicas e fragmentos em mau estado de fixação.

Com base nos critérios adotados, o número de fragmentos de autópsias coletadas e avaliadas foi de 60, divididos em 4 grupos descritos da seguinte forma: fragmentos de autópsias de adultos com a SIDA (ACA), fragmentos de autópsias de adultos sem a SIDA (ASA), fragmentos de autópsias de idosos com a SIDA (ICA) e fragmentos de autópsias de idosos sem a SIDA (ISA). O grupo sem SIDA foi formado de fragmentos de autópsias de indivíduos não infectados pelo VIH no mesmo osso, independente da causa da morte desde que não apresentassem nenhum dos critérios de exclusão ou doenças acima citadas. Ressaltamos que na literatura pacientes com SIDA é considerado idoso quando apresenta idade igual ou superior a 50 anos (Unaids, 2013; Mccomsey, et al., 2010). A caracterização dos grupos quanto à faixa etária, demonstra que para os grupos que envolviam adultos a idade definida foi inferior a 50 anos, enquanto para os grupos de idosos foi superior a 50 anos, 
conforme tabela 1. Nesse estudo também comparamos os grupos para avaliar se o fator SIDA leva a alterações predominantes em determinado grupo, assim a comparação foi feita de acordo com a faixa etária de idade de adultos e idosos e de acordo com o quadro clínico com ou sem SIDA.

Tabela 1. Caracterização das idades dos grupos.

\begin{tabular}{lll}
\hline Grupo & Idade min/máx. & Média da idade \\
ACA & $26-48$ & 43 \\
ASA & $26-49$ & 41 \\
ICA & $51-68$ & 57 \\
ISA & $60-71$ & 65 \\
\hline
\end{tabular}

Idades mínimas e máximas (min/máx.), adultos com a SIDA (ACA), adultos sem a SIDA (ASA) idosos com a SIDA (ICA) idosos sem a SIDA (ISA). Fonte arquivo próprio.

Após os critérios de seleção e realização da coleta do material, os fragmentos de medula óssea foram submetidos ao processamento histológico no laboratório de histotécnicas da disciplina de Patologia Geral da UFTM. Os cortes histológicos foram realizados com quatro micrômetros $(\mu \mathrm{m})$ de espessura por meio do micrótomo da marca MICROM modelo OUT $400 \mathrm{e}$ na capela da marca SP Labor a realização das colorações com Hematoxilina eosina (HE) e Picrosírius (PS).

As lâminas coradas pela HE foram utilizadas para a análise da espessura das trabéculas ósseas, expressa em $\mu \mathrm{m}$. Para obtenção das medidas, o fragmento em cada lâmina foi dividido em quatro campos, e em cada campo foi selecionado, de forma aleatória cinco trabéculas ósseas. Para cada trabécula óssea foi realizada cinco medidas, que estendiam do menor para o maior eixo. Totalizando assim 100 medidas por lâmina.

Para realização das morfometrias, tanto das trabéculas ósseas quanto do colágeno, foi utilizado o microscópio de luz da modelo Axion com câmera de vídeo acoplada da modelo ICc 5 da marca ZEISS, com aumento final de 400x. O colágeno total foi quantificado por meio de lâminas coradas pelo PS sob luz polarizada apresentando locais birrefringentes com cor avermelhada, identificados a partir da marcação da área dos campos selecionados por um único observador, para essas análises foi utilizado o programa Axio vision versão 4.9.1. A análise estatística foi realizada pelo software Statistica®, versão 10.0. Para variáveis com distribuição normal e variância homogênea foi utilizado o teste paramétrico " $t$ " de Student, na comparação entre dois grupos. Para os dados não paramétricos, na comparação entre os dois grupos, o teste de Mann-Whitney. Como diferenças estatisticamente significativas, foi adotado o $\mathrm{p}<0,05$. Para efeito de comparação dos grupos o valor-p (faixa etária) se refere ao nível de significância obtido por meio do teste t-Student ou Mann-Whitney na comparação entre os indivíduos adultos e idosos para cada quadro clínico. O valor-p (quadro clínico) se refere ao nível de significância obtido por meio do teste t-Student ou Mann-Whitney na comparação entre os indivíduos com e sem SIDA para cada faixa etária.

\section{Resultados}

Na comparação das trabéculas ósseas entre os indivíduos com e sem SIDA de acordo com cada faixa etária, observouse que o grupo ACA apresentou diminuição significativa na espessura das trabéculas ósseas em comparação com os ASA, ( $<<0,001)$ (Tabela 2, Figura 1) assim como ocorreu no grupo de (ICA) que apresentou diminuição significativa na espessura das trabéculas ósseas em comparação aos ISA, (p<0,001) (Tabela 2, Figura 2). Na comparação entre os indivíduos adultos e idosos segundo a presença ou não da SIDA, identificamos um aumento significativo na espessura das trabéculas ósseas no grupo de ISA em comparação com os ASA, (p<0,001) (Tabela 2). 
Tabela 2. Espessura média das trabéculas ósseas.

\begin{tabular}{llll}
\hline Espessura média das trabéculas & Adulto & Idoso & ${\text { Valor }-\mathbf{p}^{\mathbf{1}}}$ \\
\hline Com SIDA & $104,91 \pm 12,21(\mu \mathrm{m})$ & $105,94 \pm 2,11(\mu \mathrm{m})$ & 0,074 \\
Sem SIDA & $140,17 \pm 4,50(\mu \mathrm{m})$ & $152,69 \pm 5,40(\mu \mathrm{m})$ & $<\mathbf{0 , 0 0 1}$ \\
Valor $-\mathrm{p}^{\mathbf{2}}$ & $<\mathbf{0 , 0 0 1}$ & $<\mathbf{0 , 0 0 1}$ & \\
\hline
\end{tabular}

Resultados das medidas das espessuras das trabéculas ósseas dos grupos ACA, ASA, ICA e ISA. Significativo para o valor de $(\mathrm{p}<0,05) . A C A=$ adultos com SIDA, ASA=adultos sem SIDA, ICA= idosos com SIDA e ISA= idosos sem SIDA. O valor-p ${ }^{1}$ (faixa etária) se refere ao nível de significância obtido por meio do teste t-Student ou Mann-Whitney na comparação entre os indivíduos adultos e idosos com e sem SIDA. O valor-p² (quadro clínico) se refere ao nível de significância obtido por meio do teste t-Student ou Mann-Whitney na comparação entre os indivíduos com SIDA e sem SIDA para cada faixa etária. Fonte: Arquivo próprio.

Figura 1. Trabéculas ósseas dos grupos ACA e ASA.
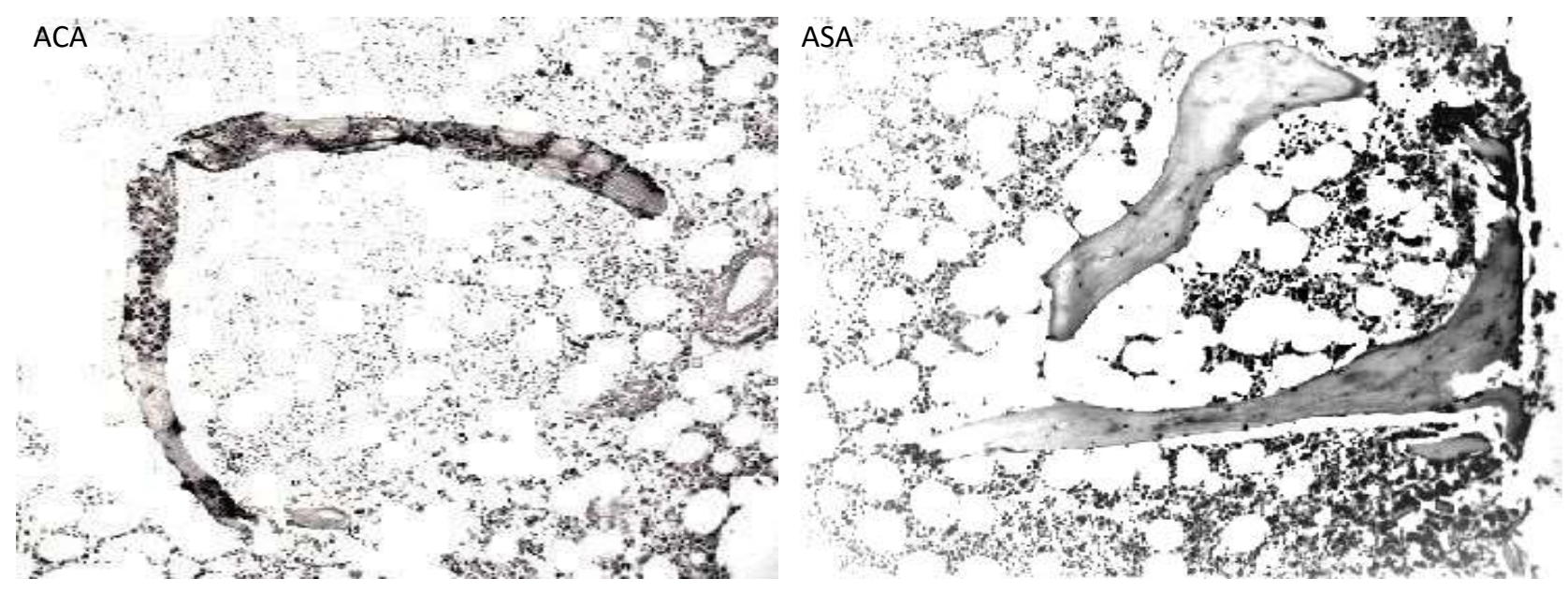

Nas duas imagens podemos observar duas trabéculas ósseas em cada grupo, sendo as do grupo ACA com espessura menor. Técnica Hematoxilina Eosina. Aumento final 400x. ACA= Adultos com SIDA, ASA= Adultos sem SIDA. Fonte Arquivo próprio. 
Figura 2. Medidas das espessuras das trabéculas ósseas dos grupos ICA e ISA.
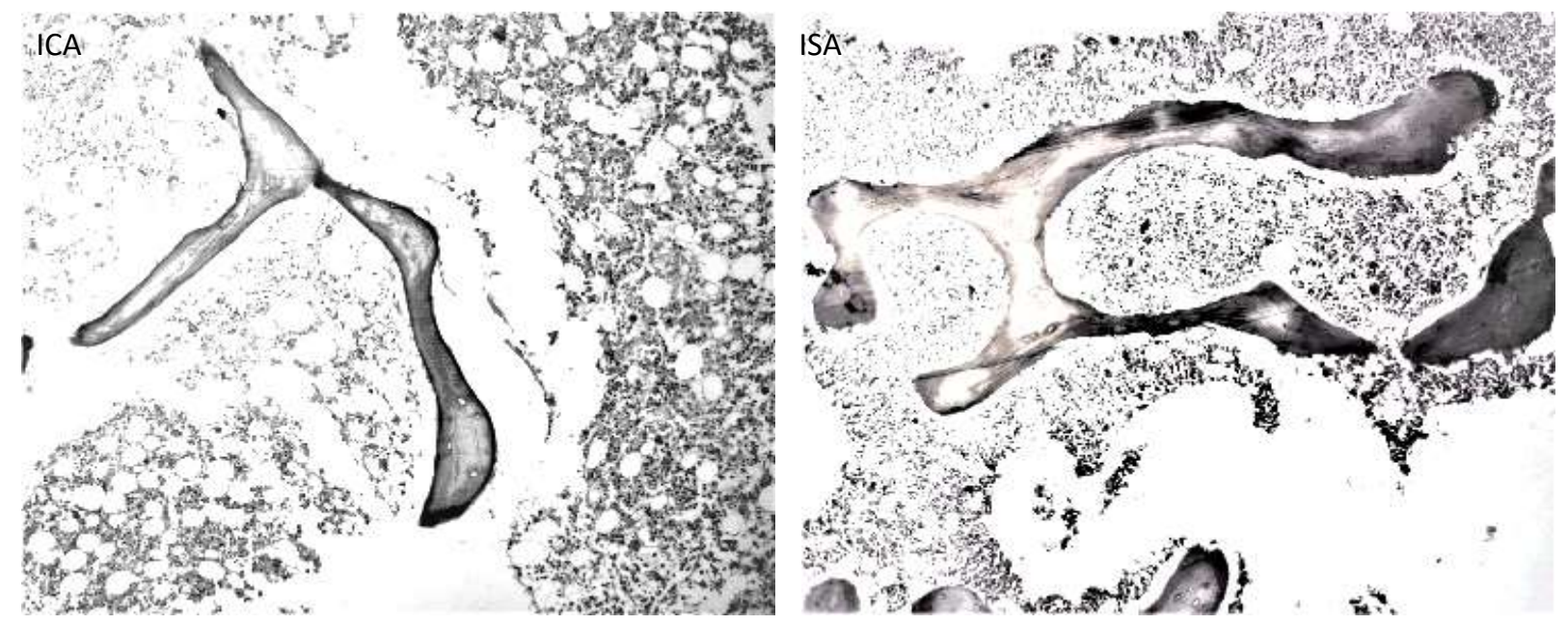

Medidas das espessuras das trabéculas ósseas dos grupos ICA e ISA com redução na espessura das trabéculas do grupo ICA. Coloração: Hematoxilina Eosina. Aumento final 400X. ICA= Idosos com SIDA, ISA= Idosos sem SIDA. Fonte Arquivo próprio.

$\mathrm{Na}$ análise do colágeno total entre os indivíduos com e sem SIDA para cada taixa etária, veriticou-se que o grupo de ACA apresentou aumento significativo do colágeno total em comparação aos ASA (p<0,001) (Tabela 3, Figura 3). Da mesma forma, foi observado que o grupo de ICA apresentou aumento significativo do colágeno total em comparação aos ISA, (p0,002) (Tabela 3, Figura 4). Na comparação entre os indivíduos adultos e idosos, de acordo com a presença ou não da SIDA, foi identificado aumento significativo do colágeno total nos ACA em comparação aos ICA (p0,041) (Tabela 3, Figura 5).

Tabela 3. Porcentagem do colágeno total das trabéculas ósseas.

\begin{tabular}{llll}
\hline Colágeno total $(\boldsymbol{\%})$ & Adulto & Idoso & Valor $^{-p^{\mathbf{1}}}$ \\
\hline Com SIDA & $27,32 \pm 15,65$ & $16,31 \pm 9,36$ & $\mathbf{0 , 0 4 1}$ \\
Sem SIDA & $8,86 \pm 11,62$ & $5,76 \pm 3,33$ & 0,814 \\
Valor $-\mathrm{p}^{\mathbf{2}}$ & $\mathbf{< 0 , 0 0 1}$ & $\mathbf{0 , 0 0 2}$ & \\
\hline
\end{tabular}

Resultados da porcentagem de colágeno total dos grupos ACA, ASA, ICA e ISA. Significativo para o valor de $(p<0,05)$. $\mathrm{ACA}=$ adultos com SIDA, ASA=adultos sem SIDA, ICA= idosos com SIDA e ISA= idosos sem SIDA. O valor- $\mathrm{p}^{1}$ (faixa etária) se refere ao nível de significância obtido por meio do teste t-Student ou Mann-Whitney na comparação entre os indivíduos adultos e idosos com ou sem SIDA. O valor-p² (quadro clínico) se refere ao nível de significância obtido por meio do teste t-Student ou Mann-Whitney na comparação entre os indivíduos com SIDA e sem SIDA para cada faixa etária. Fonte Arquivo próprio.

Figura 3. Colágeno total dos grupos ACA e ASA, com aumento significativo do colágeno no grupo de ACA. Coloração: Picrosírius sobre luz polarizada. Aumento final 400x. ACA= adultos com SIDA, ASA= adultos sem SIDA.
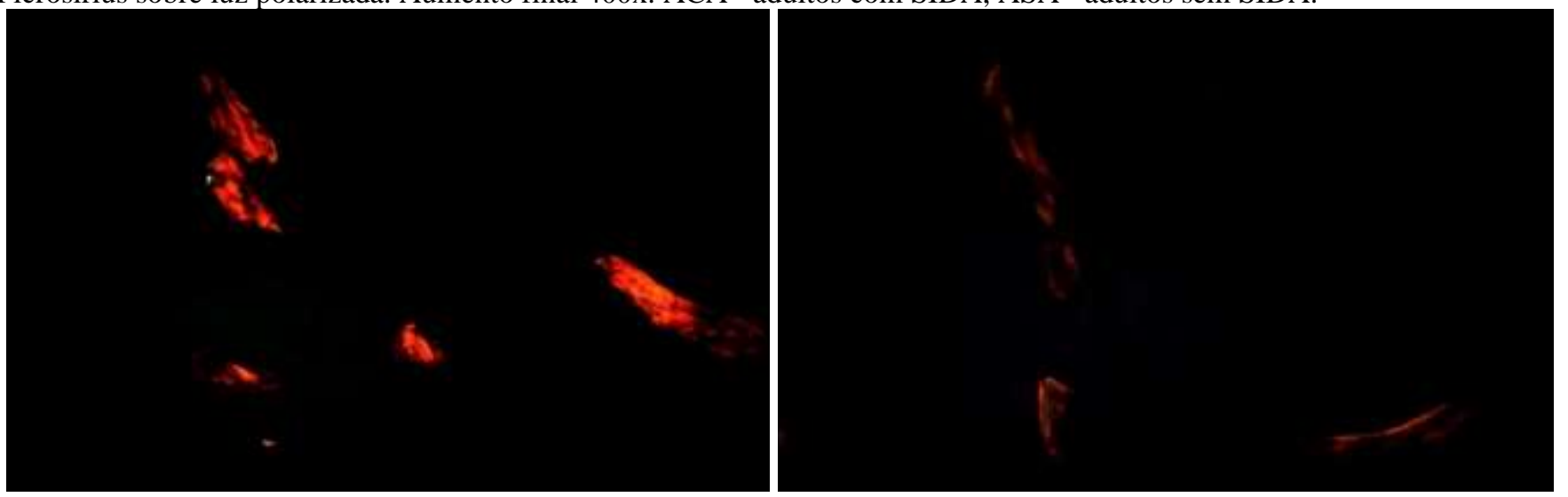

Fonte Arquivo próprio. 
Figura 4. Colágeno total dos grupos ICA e ISA, com aumento significativo do colágeno no grupo de ICA. Coloração: Picrosírius sobre luz polarizada. Aumento final 400x. ICA= Idosos com SIDA, ISA= Idosos sem SIDA.
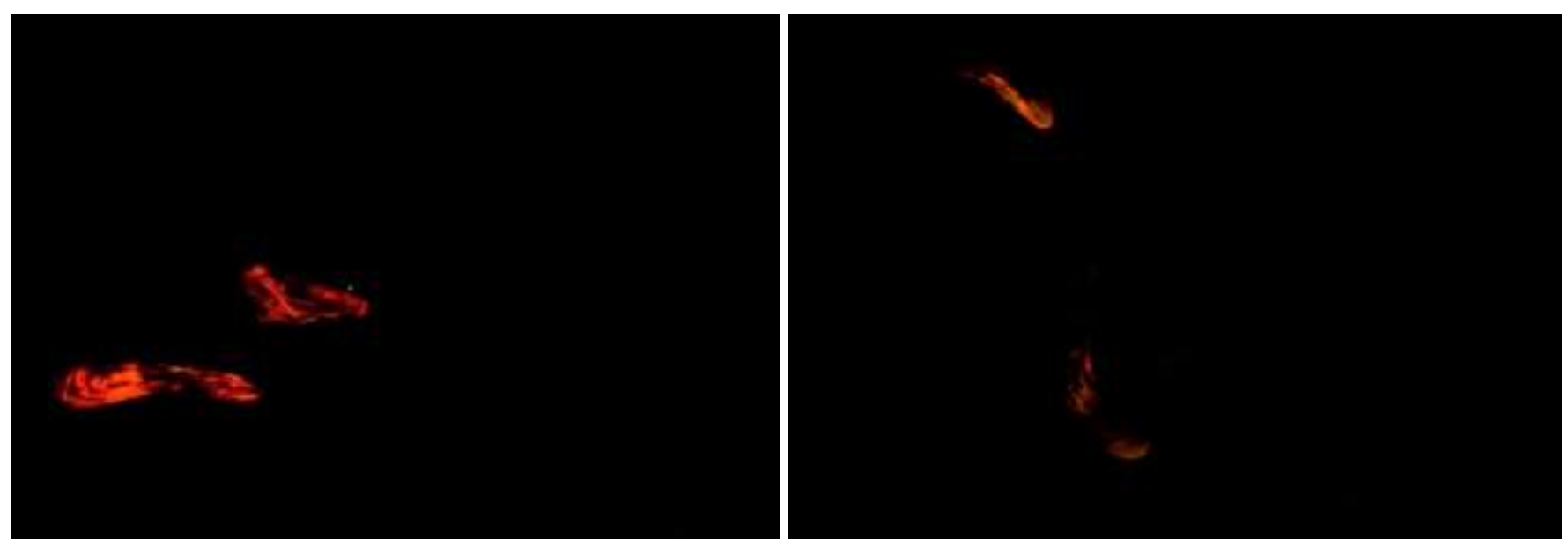

Fonte Arquivo próprio.

Figura-5. Colágeno total dos grupos ACA e ICA, com aumento significativo do colágeno no grupo de ACA. Coloração: Picrosírius sobre luz polarizada. Aumento final 100x. ACA= Adultos com SIDA, ICA= Idosos com SIDA.
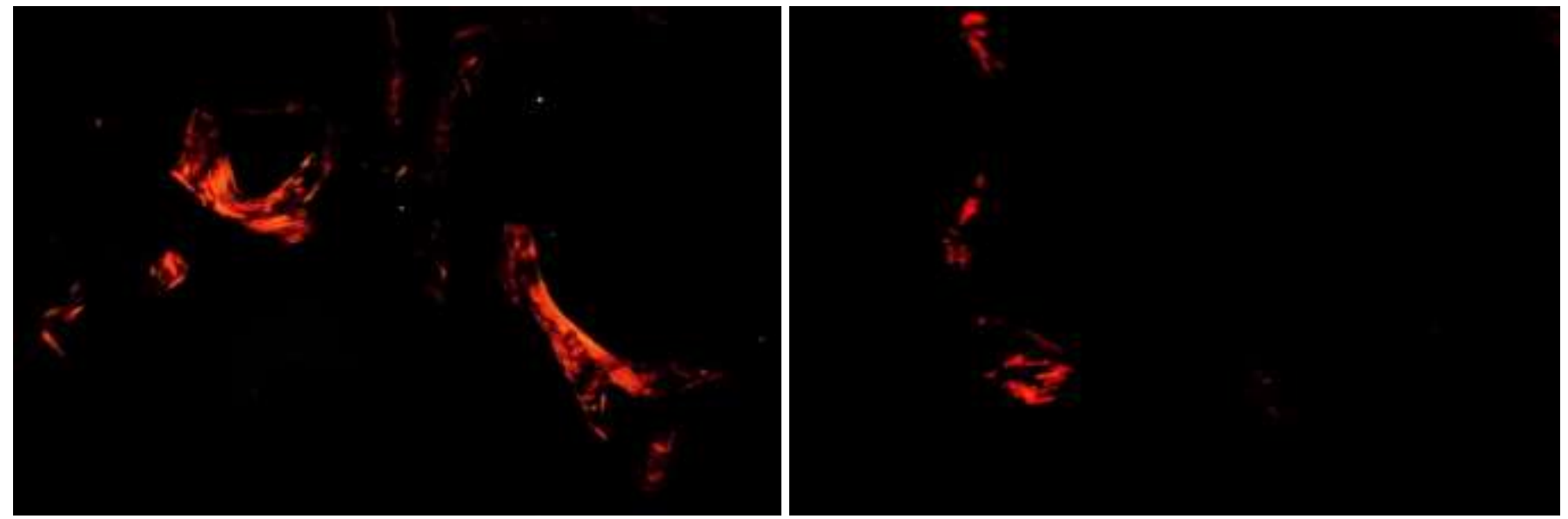

Fonte Arquivo próprio.

Verificou-se que o IMC entre os grupos de acordo com a faixa etária apresentou diferenças, porém, com diminuição significativa entre o grupo de ICA em comparação com os ACA ( $p=0,040)$. Nas demais comparações não foram observadas diferenças estatisticamente significativas (Tabela 4). 
Tabela 4. Índice de massa corporal IMC dos grupos.

\begin{tabular}{llll}
\hline IMC $\left(\mathbf{K g} / \mathbf{m}^{\mathbf{2}}\right)$ & Adulto & Idoso & ${\text { Valor }-\mathbf{p}^{\mathbf{1}}}$ \\
\hline Com SIDA & $22,71 \pm 5,35$ & $18,85 \pm 3,52$ & $\mathbf{0 , 0 4 0}$ \\
Sem SIDA & $24,75 \pm 7,35$ & $21,77 \pm 3,60$ & 0,224 \\
Valor $-\mathrm{p}^{\mathbf{2}}$ & 0,329 & 0,069 & \\
\hline
\end{tabular}

Resultados do índice de massa corporal dos grupos ACA, ASA, ICA e ISA. Significativo para o valor de $(\mathrm{p}<0,05)$. ACA=adultos com SIDA, ASA=adultos sem SIDA, ICA= idosos com SIDA e ISA=idosos sem SIDA. O valor-p ${ }^{1}$ (faixa etária) se refere ao nível de significância obtido por meio do teste t-Student ou Mann-Whitney na comparação entre os indivíduos adultos e idosos com ou sem SIDA. O valor-p² (quadro clínico) se refere ao nível de significância obtido por meio do teste t-Student ou Mann-Whitney na comparação entre os indivíduos com SIDA e sem SIDA para cada faixa etária. Fonte arquivo próprio.

Como resultado complementar apresentamos a relação da cor (tabela 5) e sexo biológico (tabela 6) com o colágeno, IMC e espessura das trabéculas ósseas, não demonstrou diferença significativa. Entretanto, esses dados fornecem características importantes para entender a distribuição de cada parâmetro estudado e sua relação com a população desse estudo.

Tabela 5. Relação da cor com os parâmetros colágeno, IMC e espessura das trabéculas ósseas.

\begin{tabular}{lccc}
\hline \multirow{2}{*}{ Parâmetros } & \multicolumn{2}{c}{ Cor } & \multirow{2}{*}{ Valor-p } \\
\cline { 2 - 3 } & Branco & Preto & \\
\hline Colágeno $(\%)$ & $15,38 \pm 15,34$ & $17,27 \pm 12,13$ & 0,684 \\
IMC $\left(\mathrm{Kg} / \mathrm{m}^{2}\right)$ & $22,75 \pm 6,20$ & $21,69 \pm 3,56$ & 0,561 \\
Espessura $(\mu \mathrm{m})$ & $126,16 \pm 22,01$ & $117,10 \pm 21,18$ & 0,192 \\
\hline
\end{tabular}

Relação da cor com os parâmetros colágeno, IMC e espessura das trabéculas ósseas dos grupos ACA, ASA, ICA e ISA. ACA=adultos com SIDA, ASA=adultos sem SIDA, ICA= idosos com SIDA e ISA=idosos sem SIDA. O valor-p ${ }^{1}$ (faixa etária) se refere ao nível de significância obtido por meio do teste t-Student ou Mann-Whitney na comparação entre os indivíduos adultos e idosos com ou sem SIDA. $\mathrm{O}$ valor-p² (quadro clínico) se refere ao nível de significância obtido por meio do teste t-Student ou Mann-Whitney na comparação entre os indivíduos com SIDA e sem SIDA para cada faixa etária. Significativo para o valor de $(\mathrm{p}<0,05)$. Fonte arquivo próprio.

Tabela 6. Relação do sexo com os parâmetros colágeno, IMC e espessura das trabéculas ósseas.

\begin{tabular}{lccc}
\hline \multirow{2}{*}{ Parâmetros } & \multicolumn{2}{c}{ Sexo } & \multirow{2}{*}{ Valor-p } \\
\cline { 2 - 3 } & Masculino & Feminino & \\
\hline Colágeno $(\%)$ & $15,06 \pm 15,05$ & $17,82 \pm 14,30$ & 0,485 \\
$\mathrm{IMC}\left(\mathrm{Kg} / \mathrm{m}^{2}\right)$ & $21,46 \pm 5,29$ & $23,93 \pm 6,16$ & 0,105 \\
Espessura $(\mu \mathrm{m})$ & $124,94 \pm 22,00$ & $122,10 \pm 22,20$ & 0,629 \\
\hline
\end{tabular}

Relação do sexo com os parâmetros colágeno, IMC e espessura das trabéculas ósseas dos grupos ACA, ASA, ICA e ISA. ACA= adultos com SIDA, ASA=adultos sem SIDA, ICA= idosos com SIDA e ISA= idosos sem SIDA. O valor-p ${ }^{1}$ (faixa etária) se refere ao nível de significância obtido por meio do teste t-Student ou Mann-Whitney na comparação entre os indivíduos adultos e idosos com ou sem SIDA. O valor-p² (quadro clínico) se refere ao nível de significância obtido por meio do teste t-Student ou Mann-Whitney na comparação entre os indivíduos com SIDA e sem SIDA para cada faixa etária. Significativo para o valor de $(\mathrm{p}<0,05)$. Fonte arquivo próprio.

\section{Discussão}

Fragmentos de autópsias de adultos e idosos com e sem SIDA foram avaliados observando a espessura das trabéculas ósseas, quantidade de colágeno total e o IMC. Com relação a espessura das trabéculas ósseas, confirmamos nossa hipótese 
demonstrando que ter SIDA é um fator que interfere na estrutura do osso trabecular capaz de diminuir sua espessura. Com relação ao colágeno demonstrando que ter SIDA é um fator que interfere em sua deposição promovendo o aumento do colágeno total. Com relação ao o IMC confirmamos nossa hipótese demonstrando que ter SIDA é um fator que interfere na redução do IMC em idosos.

A avaliação da DMO do osso trabecular por absorciometria de raio-X de dupla energia (DXA) é atualmente a técnica mais utilizada, apesar de não invasivo e de baixa exposição à radiação ao paciente apresenta limitações, como resultados abaixo do valor real em pacientes com ossos pequenos e acima do valor real em pacientes com ossos maiores (Alvarenga, et al., 2019). Assim a análise histomorfométrica realizada nesse estudo apresenta-se como um método complementar e fidedigna para a análise da espessura das trabéculas ósseas e consequentemente avaliação da massa óssea.

Estudos sugerem que a microarquitetura do osso trabecular é mais afetada em pacientes infectados pelo VIH (Yin, et. Al., 2013) a infecção pode aumentar a perda óssea diretamente devido a fatores relacionados a citocinas pró-inflamatórias (Mcginty, et al., 2019) ou indiretamente devido ao uso da TARV (Pan, et al., 2004) na literatura há relatos que o início da TARV resulta em uma redução de 2-6\% na DMO durante as primeiras 96 semanas (Brown, et al., 2009). Na literatura há relatos que o uso da zidovudina, inibidor da enzima transcriptase reversa do vírus é capaz de aumentar a ação dos osteoclastos nos seus estágios iniciais in vitro e promover osteopenia em animais (Pan, Kilby \& Mcdonald, 2006). O uso do tenofovir a 30 $\mathrm{mg} / \mathrm{kg} /$ dia também é capaz de inibir a mineralização do osso neoformado, aumentar a remodelação e influenciar na porosidade do osso em animais (Castillo, et al., 2002).

Em humanos, estudos sugerem uma prevalência maior de osteopenia e osteoporose em pacientes infectados pelo VIH recebendo TARV (Tebas, et al., 2007; Brown, et al., 2009). O fármaco o Efavirenz que faz parte da terapia de combate ao vírus, por exemplo, é capaz de interagir com as vias de produção do hormônio $\mathrm{D}$ em humanos induzindo seus precursores como CYP3A4 e CYP24 resultando em níveis reduzidos. Esse hormônio em sua forma ativa controla mais de 200 genes, incluindo genes responsáveis pela regulação da proliferação celular, diferenciação e apoptose, sendo fundamental para a prevenção da perda da massa óssea (Welz, et al., 2010; Holick, 2007). O número e a espessura das trabéculas são importantes quando se considera a mecânica óssea geral porque alterações afetam a conectividade da rede trabecular, perder trabéculas tem efeito um efeito maior no enfraquecimento da rede e a diminuição da espessura acelera esse processo, na literatura há relatos de fraturas em mulheres devido a perda do número de trabéculas e em homens ocorrendo principalmente devido a diminuição da espessura (Silva, et al.,1997; Snyder, et al., 1993).

A diminuição significativa na espessura das trabéculas ósseas encontradas no estudo questão referente ao grupo ASA em comparação ao grupo ISA não corroboram com a literatura que evidencia a diminuição da massa óssea em idosos (Zelenka, et al., 2018). Tal fato pode ser explicado a partir das características da amostra estudada como por exemplo, indivíduos idosos mais ativos fisicamente e com bons hábitos alimentares.

O osso é um material composto, sofisticado, com relações entre mineral e colágeno que influenciam a resistência óssea, quanto menor a espessura das trabéculas maiores as chances da perda da quantidade delas e da conectividade da rede trabecular, tais mudanças podem afetar diretamente o volume ósseo do osso trabecular e assim comprometer as propriedades mecânicas do osso propiciando fragilidade óssea. Knott e colaboradores sugeriram que a osteoporose em ossos de animais não é apenas uma simples perda de matriz inorgânica, mas envolve mudanças significativas na bioquímica e consequentemente nas propriedades físicas da fibra de colágeno (Knott, et al., 1995). Na literatura há relatos de várias anormalidades na medula óssea, entre elas, fibrose e alteração do seu conteúdo total associado à infecção por VIH (Karcher \& Frost, 1991; Dias, et al., 2015). O estudo de Viguet e colaboradores investigou a relação do osso trabecular e do colágeno em humanos sem SIDA, demonstrando que modificações nos padrões de ligações cruzadas do colágeno pode influenciar o comportamento mecânico do osso (Viguet, et al., 2010). 
A idade é um fator a ser observado em pacientes com SIDA, pessoas com VIH podem ter doenças não transmissíveis mais precocemente que pessoas não infectadas, incluindo doença renal, cardiovasculares e câncer de pulmão não relacionados ao tabagismo (Triant, et al., 2007; Kirk, et al., 2007; Odden, et al., 2007). As comorbidades torna o cuidado complicado e a combinação da TARV com outros medicamentos aumenta significativamente a chance de interações medicamentosas potencialmente graves, que podem levar a toxicidade da droga, perda da eficácia do medicamento coadministrado e aumento da carga viral levando a uma infecção mais agressiva (Marzolini, et al., 2011). Nesse estudo a SIDA foi um fator importante para alterações da qualidade óssea nos indivíduos com mais de 50 anos, nossos dados corroboram com a literatura que evidencia que ossos humanos são mais propensos deterioração com o aumento da idade em indivíduos sem VIH (Wang, et al., 2002) e que a SIDA pode acelerar o processo de perda da massa óssea em idosos mesmo controlando fatores de risco, como peso e tabagismo (Cotter, et al., 2014; Mazzotta, et al., 2015).

Os resultados de um menor IMC no grupo de idosos em comparação ao grupo de adultos com SIDA identificados em nosso estudo também corrobora com a literatura que evidencia que pessoas com VIH que possuem baixo peso tem uma prevalência maior de DMO baixa e risco de fratura 1,9 vezes maior, em comparação com a população em geral (Mccomsey, et al., 2010; Pramukti, et al., 2020), o baixo peso está correlacionado ao quadro de osteoporose e o ganho de peso parece proteger homens e mulheres contra a perda óssea (Hannan, et al., 2000; Wu, et al., 2002). O baixo peso corporal em pacientes infectados pelo VIH apresenta várias condições como doenças preexistentes e adquiridas, quadro de virulência, exposição ao tabagismo, prática de atividade físicas, alimentação entre outras (Mccomsey, et al., 2010).

Evidenciamos em nosso estudo que a SIDA é um fator para alterações hismofométricas da medula óssea, promovendo alterações da quantidade de colágeno total e diminuição da espessura das trabéculas ósseas. Os mecanismos para elucidar os fatores relacionados são temas para estudos posteriores, como por exemplo a identificação do número total de trabéculas ósseas, associada a quantificação de sua espessura e o tipo de colágeno com técnica de imunohistoquímica. Nesse contexto, nossos achados juntamente com pesquisas futuras se tornam mais profundas e complexas a fim de fortalecer e disseminar o conhecimento acerca das alterações desencadeadas pela SIDA e contribuir para qualidade de vida desses indivíduos quanto aos aspectos ósseos.

\section{Conclusão}

Os resultados obtidos no presente estudo envolvendo fragmentos de autópsias de medula óssea de indivíduos com ou sem SIDA nos permite concluir que a presença da SIDA é um fator que influencia significativamente a diminuição da espessura das trabéculas ósseas do osso esterno em adultos e idosos, o fator idade e a presença da SIDA observado no grupo ICA não interferem significativamente na espessura das trabéculas ósseas do osso esterno quando comparado ao grupo de adultos com SIDA, a presença da SIDA é um fator que influencia significativamente o aumento do colágeno total das trabéculas ósseas do osso esterno em adultos e idosos, o fator idade e a presença da SIDA observado no grupo ICA influenciam significativamente a redução do colágeno total nas trabéculas ósseas do osso esterno quando comparado ao grupo de adultos com SIDA, o fator idade e a presença da SIDA observado no grupo ICA influenciam significativamente a redução do IMC quando comparado ao grupo de adultos com SIDA, a presença da SIDA não influencia significativamente na redução do IMC em adultos e idosos em comparação com adultos e idosos sem SIDA e a presença da SIDA é um fator de risco importante para saúde óssea de adultos e idosos.

\section{Limitações do estudo}

Como limitações do presente estudo destacamos que a pesquisa foi restrita as informações contidas nos prontuários e assim não foi possível determinar a influência de outras variáveis de doença e tratamento como o uso da TARV nesse estudo. 


\section{Agradecimentos}

Os autores agradecem pelo apoio do Conselho Nacional de Desenvolvimento Científico e Tecnológico para a Melhoria de Pessoal de Nível Superior (CNPq), a Fundação de Ensino e Pesquisa do Estado de Minas Gerais (FAPEMIG), a Fundação de Ensino e Pesquisa de Uberaba (FUNEPU), as Disciplinas de Anatomia Humana e Patologia Geral da Universidade Federal do Triângulo Mineiro e a Coordenação de Aperfeiçoamento de Pessoal de Nível Superior (CAPES).

\section{Referências}

Alvarenga, P., Silva, B. C., Diniz, M. P., Leite, M. B., Silva, C. A. M., Eleutério, J. C. M., Soares, M. M. S., Bilezikian, J. P., \& Camargos, B. M. (2019). Trabecular bone score: a useful clinical tool for the evaluation of skeletal health in women of short stature. Endocrine, 66(2), 398-404. https://doi.org/10.1007/s12020-019-02002-y.

Baig, M. A., \& Bacha, D. (2021) Histology, Bone. StatPearls Publishing.

Brown, T. T., McComsey, G. A., King, M. S., Qaqish, R. B., Bernstein, B. M., \& Silva, B. A. (2009). Loss of bone mineral density after antiretroviral therapy initiation, independent of antiretroviral regimen. Journal of acquired immune deficiency syndromes (1999), 51(5), 554-561. https://doi.org/10.1097/QAI.0b013e3181adce44.

Castillo, A. B., Tarantal, A. F., Watnik, M. R., \& Martin, R. B. (2002). Tenofovir treatment at $30 \mathrm{mg} / \mathrm{kg} / \mathrm{day}$ can inhibit cortical bone mineralization in growing rhesus monkeys (Macaca mulatta). Journal of orthopaedic research: official publication of the Orthopaedic Research Society, $20(6), 1185-1189$. https://doi.org/10.1016/S0736-0266(02)00074-8.

Clarke B. (2008). Normal bone anatomy and physiology. Clinical journal of the American Society of Nephrology: CJASN, 3 Suppl 3(Suppl 3), S131-S139. https://doi.org/10.2215/CJN.04151206.

Cotter, A. G., Sabin, C. A., Simelane, S., Macken, A., Kavanagh, E., Brady, J. J., McCarthy, G., Compston, J., Mallon, P. W., \& HIV UPBEAT Study Group (2014). Relative contribution of HIV infection, demographics and body mass index to bone mineral density. AIDS (London, England), 28(14), 2051-2060. https://doi.org/10.1097/QAD.0000000000000353.

Dias, N. F., Juliano, G. R., Espindula, A. P., de Oliveira, F. A., Oliveira, L. F., Cavellani, C. L., Ramalho, L. S., Teixeira, V., \& Ferraz, M. L. (2015). Influence of AIDS in collagen deposition and thickness of the bone marrow. Annals of diagnostic pathology, 19(6), 409-413. https://doi.org/10.1016/j.anndiagpath.2015.10.003.

GOTTLIEB, M.S. (2021) Centers For Disease Control. Pneumocystis Pneumonia, Morbidity and Mortality Weekly Report (MMWR) https://www.cdc.gov/mmwr/preview/mmwrhtml/june_5.htm.

Holick M. F. (2017). The vitamin D deficiency pandemic: Approaches for diagnosis, treatment and prevention. Reviews in endocrine \& metabolic disorders, 18(2), 153-165. https://doi.org/10.1007/s11154-017-9424-1.

Karcher, D. S., \& Frost, A. R. (1991). The bone marrow in human immunodeficiency virus (HIV)-related disease. Morphology and clinical correlation. American journal of clinical pathology, 95(1), 63-71. https://doi.org/10.1093/ajcp/95.1.63.

Kaslow, R. A., Ostrow, D. G., Detels, R., Phair, J. P., Polk, B. F., \& Rinaldo, C. R., Jr. (1987, Aug). The Multicenter AIDS Cohort Study: rationale, organization, and selected characteristics of the participants. Am J Epidemiol, 126(2), 310-318. https://doi.org/10.1093/aje/126.2.310

Kirk, G. D., Merlo, C., O' Driscoll, P., Mehta, S. H., Galai, N., Vlahov, D., Samet, J., \& Engels, E. A. (2007). HIV infection is associated with an increased risk for lung cancer, independent of smoking. Clinical infectious diseases: an official publication of the Infectious Diseases Society of America, 45(1), 103110. https://doi.org/10.1086/518606.

Knott, L., Whitehead, C. C., Fleming, R. H., \& Bailey, A. J. (1995). Biochemical changes in the collagenous matrix of osteoporotic avian bone. The Biochemical journal, 310 (Pt 3) (Pt 3), 1045-1051. https://doi.org/10.1042/bj3101045.

Marzolini, C., Back, D., Weber, R., Furrer, H., Cavassini, M., Calmy, A., Vernazza, P., Bernasconi, E., Khoo, S., Battegay, M., Elzi, L., \& Swiss HIV Cohort Study Members (2011). Ageing with HIV: medication use and risk for potential drug-drug interactions. The Journal of antimicrobial chemotherapy, 66(9), 2107-2111. https://doi.org/10.1093/jac/dkr248.

Mazzotta, E., Ursini, T., Agostinone, A., Di Nicola, A. D., Polilli, E., Sozio, F., Vadini, F., Pieri, A., Trave, F., De Francesco, V., Capasso, L., Borderi, M., Manzoli, L., Viale, P., \& Parruti, G. (2015). Prevalence and predictors of low bone mineral density and fragility fractures among HIV-infected patients at one Italian center after universal DXA screening: sensitivity and specificity of current guidelines on bone mineral density management. AIDS patient care and STDs, 29(4), 169-180. https://doi.org/10.1089/apc.2014.0205.

McComsey, G. A., Tebas, P., Shane, E., Yin, M. T., Overton, E. T., Huang, J. S., Aldrovandi, G. M., Cardoso, S. W., Santana, J. L., \& Brown, T. T. (2010). Bone disease in HIV infection: a practical review and recommendations for HIV care providers. Clinical infectious diseases: an official publication of the Infectious Diseases Society of America, 51(8), 937-946. https://doi.org/10.1086/656412.

McGinty, T., Cotter, A. G., Sabin, C. A., Macken, A., Kavanagh, E., Compston, J., Sheehan, G., Lambert, J., Mallon, P., \& HIV UPBEAT (Understanding the Pathology of Bone Diseases in HIV-infected Subjects) Study Group (2019). Assessment of trabecular bone score, an index of bone microarchitecture, in HIV positive and HIV negative persons within the HIV UPBEAT cohort. PloS one, 14(3), e0213440. https://doi.org/10.1371/journal.pone.0213440.

Odden, M. C., Scherzer, R., Bacchetti, P., Szczech, L. A., Sidney, S., Grunfeld, C., \& Shlipak, M. G. (2007). Cystatin C level as a marker of kidney function in human immunodeficiency virus infection: the FRAM study. Archives of internal medicine, 167(20), 2213-2219. https://doi.org/10.1001/archinte.167.20.2213. 
Odden, M. C., Scherzer, R., Bacchetti, P., Szczech, L. A., Sidney, S., Grunfeld, C., \& Shlipak, M. G. (2007). Cystatin C level as a marker of kidney function in human immunodeficiency virus infection: the FRAM study. Archives of internal medicine, 167(20), 2213-2219. https://doi.org/10.1001/archinte.167.20.2213.

Pan, G., Wu, X., McKenna, M. A., Feng, X., Nagy, T. R., \& McDonald, J. M. (2004). AZT enhances osteoclastogenesis and bone loss. AIDS research and human retroviruses, 20(6), 608-620. https://doi.org/10.1089/0889222041217482.

Secretaria de Vigilância em Saúde. Boletim Epidemiológico HIV/Aids 2020: Boletim de aids e dst HIV/AIDS. http://www.aids.gov.br/ptbr/pub/2020/boletim-epidemiologico-hivaids-2020.

Silva, A. C. (2019) Tese Doutorado no programa de pós graduação em ciências da saúde UFTM. Análise das alterações hepáticas em pacientes autopsiados com a síndrome da imunodeficiência adquirida.

Silva, M. J., \& Gibson, L. J. (1997). Modeling the mechanical behavior of vertebral trabecular bone: effects of age-related changes in microstructure. Bone, 21(2), 191-199. https://doi.org/10.1016/s8756-3282(97)00100-2.

Snyder, B. D., Piazza, S., Edwards, W. T., \& Hayes, W. C. (1993). Role of trabecular morphology in the etiology of age-related vertebral fractures. Calcified tissue international, 53 Suppl 1, S14-S22. https://doi.org/10.1007/BF01673396.

Starup-Linde, J., Rosendahl, S. B., Storgaard, M., \& Langdahl, B. (2020). Management of Osteoporosis in Patients Living With HIV-A Systematic Review and Meta-analysis. Journal of acquired immune deficiency syndromes (1999), 83(1), 1-8. https://doi.org/10.1097/QAI.0000000000002207.

Tebas, P. (2007) Initiation of ART is associated with bone loss independent of the specific ART regimen: results of ACTG A5005s. Presented at: 14th Conference on Retroviruses and Opportunistic Infections.

Triant, V. A., Lee, H., Hadigan, C., \& Grinspoon, S. K. (2007). Increased acute myocardial infarction rates and cardiovascular risk factors among patients with human immunodeficiency virus disease. The Journal of clinical endocrinology and metabolism, 92(7), 2506-2512. https://doi.org/10.1210/jc.2006-2190.

UNAIDS. (2013). Global report: Unaids report on the global aids epidemic 2013. https://www.unaids.org/en.

UNAIDS. Unaids data 2020. https://www.unaids.org/sites/default/files/media_asset/2020_aids-data-book_en.pdf.

Viguet Carrin, S., Follet, H., Gineyts, E., Roux, J. P., Munoz, F., Chapurlat, R., Delmas, P. D., \& Bouxsein, M. L. (2010). Association between collagen crosslinks and trabecular microarchitecture properties of human vertebral bone. Bone, 46(2), 342-347. https://doi.org/10.1016/j.bone.2009.10.001.

Wang, X., Shen, X., Li, X., \& Agrawal, C. M. (2002). Age-related changes in the collagen network and toughness of bone. Bone, 31(1), 1-7. https://doi.org/10.1016/s8756-3282(01)00697-4.

Welz, T., Childs, K., Ibrahim, F., Poulton, M., Taylor, C. B., Moniz, C. F., \& Post, F. A. (2010). Efavirenz is associated with severe vitamin D deficiency and increased alkaline phosphatase. AIDS (London, England), 24(12), 1923-1928. https://doi.org/10.1097/QAD.0b013e32833c3281.

Yarchoan, R., \& Uldrick, T. S. (2018). HIV-Associated Cancers and Related Diseases. The New England journal of medicine, 378(11), 1029-1041. https://doi.org/10.1056/NEJMra1615896.

Yin, M. T., Shu, A., Zhang, C. A., Boutroy, S., McMahon, D. J., Ferris, D. C., Colon, I., \& Shane, E. (2013). Trabecular and cortical microarchitecture in postmenopausal HIV-infected women. Calcified tissue international, 92(6), 557-565. https://doi.org/10.1007/s00223-013-9716-8.

Zaidi, M. et al. Encyclopedia of Bone Biology: anatomy and structural considerations. Ed. Academic Press, $218-232$.

Zelenka, L., Alt, J., Knížková, I., Kunc, P., \& Lukešová, D. (2018). Epidemiologická studie vlivu pohlaví, věku, mobility a doby úrazu na zlomeniny proximálního femuru [Epidemiological Study of the Effects of Gender, Age, Mobility and Time of Injury on Proximal Femoral Fractures]. Acta chirurgiae orthopaedicae et traumatologiae Cechoslovaca, 85(1), 40-45. 